\title{
Gentamicin-loaded discs and microspheres and their modifications: characterization and in vitro release
}

\author{
Pavan Kumar Naraharisetti ${ }^{\mathrm{a}}$, Magdeleine Duan Ning Lew ${ }^{\mathrm{a}}$, Yin-Chih Fu ${ }^{\mathrm{b}}$, \\ Duu-Jong Lee ${ }^{\mathrm{c}}$, Chi-Hwa Wang ${ }^{\mathrm{a}, \mathrm{d}, *}$ \\ ${ }^{a}$ Department of Chemical and Biomolecular Engineering, National University of Singapore, 4 Engineering Drive 4, \\ Singapore 117576, Singapore \\ ${ }^{\mathrm{b}}$ Department of Orthopaedic Surgery, Kaohsiung Medical University Hospital, 100 Shith-Chuan 1st Road, Kaohsiung 807, Taiwan \\ ${ }^{c}$ Department of Chemical Engineering, National Taiwan University, No 1, Section 4, Roosevelt Road, Taipei 106, Taiwan \\ ${ }^{\mathrm{d}}$ Molecular Engineering of Biological and Chemical Systems, Singapore-MIT Alliance, Singapore 117576, Singapore
}

Received 15 September 2003; accepted 14 October 2004

Available online 14 November 2004

\begin{abstract}
Osteomyelitis is an infection of the bone, and successful treatment involves local administration for about 6 weeks. Gentamicin is a very hydrophilic drug and tends to come out into the water phase when microspheres are fabricated using solvent evaporation method. Hence, spray drying is an option, and it was observed that the release rate tends to be fast when the particle size is small and large particles cannot be prepared by spray drying. In an effort to get better encapsulation efficiency and release rate, we have worked on the possibility of compressing the microspheres into discs and modifying the porosity of the discs by using biocompatible materials like polyethylene glycol (PEG) and calcium phosphates and also on the fabrication of double-walled and composite microspheres. In the case of microspheres, two methods of fabrication both based on solvent evaporation method were employed. The two polymers used are poly-L-lactide (PLLA) and copolymers of polyDL-lactic-co-glycolic acid (PLGA). One method is based on the spreading coefficient theory for the formation of doublewalled microspheres by using single solvent, while the other is based on the property of PLLA not being soluble in ethyl acetate (EA). Characterization to check if the microspheres formed are double-walled was performed. The fabrication method where two solvents, dichloromethane (DCM) and ethyl acetate, were used gave double-walled microspheres, while the other where only dichloromethane was used gave composites. The double-walled microspheres were smaller in size compared to the composites, which were in the range of 100-600 $\mu \mathrm{m}$. This can be attributed to the difference in the fabrication procedure. We were able to achieve better encapsulation efficiencies of more than $50 \%$ and slower release rates, which lasted for about 15 days. It was observed that size played a major role in the encapsulation efficiency and release rates. The possibility of
\end{abstract}

* Corresponding author. Department of Chemical and Biomolecular Engineering, National University of Singapore, 4 Engineering Drive 4 , Singapore 117576, Singapore. Tel.: +65 68745079; fax: +65 67791936 .

E-mail address: chewch@nus.edu.sg (C.-H. Wang). 
achieving better results by studying the effect of concentration of polymer in solvent and the effect of using different polymers was investigated.

(C) 2004 Elsevier B.V. All rights reserved.

Keywords: Disc; Double-walled; Microspheres; Gentamicin; Biodegradable

\section{Introduction}

There are many methods for the prevention and treatment of bone infection of which prolonged systemic antibiotic therapy for 4-6 weeks, and local implantation of the nonbiodegradable polymer polymethyl methacryllate (PMMA) containing Gentamicin is common. Each of the methods has their own disadvantages. By systemic therapy, only a small fraction of the dose reaches the surgical site, producing low therapeutic tissue levels and often various adverse side effects, including the development of bacterial resistance to drugs. Local administration of PMMA beads has been employed clinically to prevent or treat Osteomyelitis. PMMA, however, is a nonbiodegradable material, and secondary, surgery is required to remove the beads after the Gentamicin is released [1].

Great effort has been made in recent years to obtain biodegradable synthetic polymer implants for treating Osteomyelitis. PLA and poly-DL-lactic-co-glycolic acid (PLGA) have shown sufficient absorption, biocompatibility, mechanical properties and positive effects on new bone formation for treating bonerelated problems. PLA, PLGA and other biocompatible and biodegradable polymers such as polyanhydrides and polyhydroxybutyrate-co-hydroxyvalerate (PHBV) have been used to prepare bone implants with different antibiotics. These results in different animal species show the effectiveness of these materials as antibiotic carrier for bone implantation in the treatment of local infections. All these systems have the advantage over PMMA beads that no second surgical procedure is required for implant removal, along with certain osteoregenerative properties [2-18].

It was observed during the in vitro release from the discs made of PLGA microspheres (that were prepared by spray drying) that they tend to give a biphasic release based on the amount of drug that is present near the surface of the drug and the bulk degradation of the polymer. Hence, we have worked on the possibility of modifying the porosity of the discs by the addition of other biocompatible materials like polyethylene glycol (PEG) and calcium phosphates, which help in increasing the porosity of the disc and hence eliminating the lag phase that is observed in the pure discs. We have also worked on fabrication, characterization and in vitro release of the antibiotic from biodegradable polymers and their blends by two other fabrication methods [19-28]. First, the fabrication of microspheres of PLGA-polyL-lactide (PLLA) is done using a single solvent phase separation method. The second is based on two solvents where one of the polymers is soluble in only one of the solvent, whereas the other polymer is soluble in both the solvents. Our effort was directed towards the fabrication of double-walled microspheres. The characterization to check if doublewalled microspheres are formed, and in vitro release study was carried out. The fabrication of doublewalled microspheres gave better results in terms of encapsulation efficiency and release time, and using a single polymer often tends to little encapsulation efficiency because Gentamicin is hydrophilic. Also, if spray drying is employed, then the size of the particle tends to be small and the release may be faster. Since, two methods were employed for the fabrication of microspheres and only one of them gave doublewalled microspheres, the presentation on microspheres is presented in two parts. The discs and their modifications are presented as Part A, composite microspheres are presented as Part B and the doublewalled microspheres as Part $\mathrm{C}$ of the study for easier understanding.

\section{Materials and methods}

\subsection{Materials}

Poly(DL-lactic-co-glycolic acid 50:50) $\left(\mathrm{M}_{\mathrm{w}} 40,000-\right.$ $75,000)$, poly(DL-lactic-co-glycolic acid 65:35) $\left(\mathrm{M}_{\mathrm{w}}\right.$ 
Table 1

Seven samples (A-G) with different percentages of PEG and different molecular weights of PEG were used to prepare the microspheres

\begin{tabular}{ll}
\hline Sample & EE \% \\
\hline A & $62.1 \pm 1.9$ \\
B & $61.7 \pm 2.9$ \\
C & $62.4 \pm 2.2$ \\
D & $45.5 \pm 2.6$ \\
E & $42.6 \pm 2.0$ \\
F & $51.4 \pm 2.4$ \\
G & $49.4 \pm 2.1$ \\
\hline
\end{tabular}

A-2\% PEG (mol. wt. 8000); B-5\% PEG (mol. wt. 8000); C$10 \%$ PEG (mol. wt. 8000); D-15\% PEG (mol. wt. 8000); E-20\% PEG (mol. wt. 8000); F-10\% PEG (mol. wt. 3350); G-10\% PEG (mol. wt. 1450). For all samples, PLGA 50:50 was used as the main polymer along with PEG.

40,000-75,000), poly(DL-lactic-co-glycolic acid 85: 15) Gentamicin sulfate USP, $O$-phthalaldehyde, $O$ phthaldialdehyde, boric acid, 2-mercaptoethanol, methylcellulose (MC), polyethylene glycol mol. wt. 1450, polyethylene glycol mol. wt. 3350, polyethylene glycol mol. wt. 8000, polyvinyl alcohol (PVA) mol. wt. 30,000-70,000, phosphate buffer saline (PBS; $0.138 \mathrm{M} \mathrm{NaCl}, 0.0027 \mathrm{M} \mathrm{KCl}, 0.01 \mathrm{M}$ phosphate buffer saline) used for in vitro release study were all purchased from Sigma-Aldrich (St. Louis, USA). Ethyl acetate (EA), dichloromethane (DCM) and methanol are from Tedia (Fairfield, $\mathrm{OH}$, USA). Hydroxylapatite is from Raedel-de-Haen, Seelze, Germany. Beta-tricalcium phosphate is from Fluka (Steinheim, Switzerland). Sodium hydroxide and acetic acid are from Merck (Darmstadt, Germany). 1-heptane sulphonic acid sodium salt is from Acros (New Jersey, USA) and Aldrich (Steinheim, Germany).

\subsection{Methods}

\subsubsection{Part A}

One gram of the required polymer is dissolved in $50 \mathrm{ml}$ of ethyl acetate (if two types of polymers are used, then the total weight of polymer is $1 \mathrm{~g}$ ). If polyethylene glycol (PEG) or methylcellulose (MC) is used, then dichloromethane (DCM) is used as solvent, as PEG is not soluble in ethyl acetate. MC can only be suspended in the dichloromethane solution (the solution turns turbid), and it cannot be suspended in ethyl acetate. The Gentamicin being used is dissolved in $1 \mathrm{ml}$ of water and poured into the polymer solution. The mixture is ultrasonicated at 15 W for three times, for $20 \mathrm{~s}$ each time (Misonix, XL 2000, USA). The mixture is then spray dried in Buchi 191 Mini Spray Drier (Flawil, Switzerland) at $70{ }^{\circ} \mathrm{C}$, airflow rate of $700 \mathrm{Nl} / \mathrm{h}$; pump feed rate of $30 \%$ and an aspirator ratio of $100 \%$. The outlet temperature was observed to be between 45 and $50{ }^{\circ} \mathrm{C}$. The microparticles obtained were freeze-dried (Christ Alpha 1-2 Model 100200, Germany) over night for the elimination of the solvents and water that may be present. The particles were then stored in a dessicator for later use (Tables 1 and 2 present various compositions). Discs of 5-mm diameter are prepared by compressing $25 \mathrm{mg}$ of the microspheres (either those made of pure PLGA or those modified with MC and PEG) in a hydraulic hand press at a pressure of 2 tons (Graseby Specac, Orpington Kent, Britain). A pressure of 2 tons is selected, as an increase in pressure to 5 tons does not change the in vitro release profile and 2 tons pressure is well above critical limit [20]. There is no effect of pressure above a certain critical pressure. However, it is obvious that the same cannot be said when pressure is decreased below a critical value.

The fabrication method for part B is based on the relative immiscibility of polymer solutions at high concentrations. For that of part $\mathrm{C}$, the property of PLLA not being soluble in ethyl acetate (EA) is utilized.

\subsubsection{Part B}

Typically, $12 \mathrm{mg}$ of GS was suspended in dichloromethane (DCM) by sonication for $20 \mathrm{~s}$ at $20 \mathrm{~W}$ (Misonix, XL2000) so as to break down Gentamicin into smaller fragments and achieve better encapsulation and uniformity, and then PLGA was added and allowed to dissolve. PLLA-DCM solution is prepared

Table 2

Encapsulation efficiency for methylcellulose-PLGA microparticles

\begin{tabular}{ll}
\hline Sample & EE \% \\
\hline MC 2\% & $54.8 \pm 3.2$ \\
MC 5\% & $50.3 \pm 3.5$ \\
MC $10 \%$ & $45.8 \pm 4.3$ \\
\hline
\end{tabular}

$\mathrm{MC}$ is methylcellulose, and the number represents the percentage of $\mathrm{MC}$ in total weight of polymers. For all samples, PLGA 50:50 was used as the main polymer. 
simultaneously, and the PLGA solution is added and sonicated again. Subsequently, the mixture was added to $2 \%(\mathrm{w} / \mathrm{v})$ of $500 \mathrm{ml} \mathrm{PVA}$ and stirred at $400 \mathrm{rpm}$ (IKA Eurostar TD, Germany) for $4 \mathrm{~h}$, washed, filtered using a $0.8-\mu \mathrm{m}$ membrane and freeze dried (Christ Alpha1-2 Freeze Drier Model 100200$)$. The resulting microspheres were stored in dessicator for later use. Samples C2 and C3 are different (Table 3), in that for $\mathrm{C} 2$, drug was loaded into PLGA phase, and for C3, the drug was loaded into PLLA phase (instead of PLGA solution, as described above) during fabrication procedure.

\subsubsection{Part $C$}

Typically, $12 \mathrm{mg}$ of GS was suspended in DCM by sonication, and PLLA was added to this. This was added to PLGA-EA and sonicated again. This solution is then added to PVA solution (same as above). In this case, the concentration of PLLA in DCM was kept relatively higher compared to the concentration of PLGA in EA so as to achieve better encapsulation efficiency. The different PLGA-DCM concentration or PLGA-EA concentration, PLLADCM concentration and PLLA to PLGA ratio are listed in Table 3. The total mass of polymer was always $300 \mathrm{mg}$.

\subsection{Encapsulation efficiency, particle size and sur- face chemistry}

Five milligrams of the microspheres are dissolved in DCM. Five milliliter of water is added to this, vortexed and shaken to extract the Gentamicin to water phase. The supernatant water is analyzed for

Table 3

Different compositions and types of PLGA that are used in the study

\begin{tabular}{lllll}
\hline Sample & $\begin{array}{l}\text { PLGA/PLLA } \\
\text { (ratio) }\end{array}$ & $\begin{array}{l}\text { PLLA/DCM } \\
(\mathrm{w} / \mathrm{v} \%)\end{array}$ & $\begin{array}{l}\text { PLGA/DCM } \\
(\mathrm{w} / \mathrm{v} \%\end{array}$ & $\begin{array}{l}\text { PLGA/EA } \\
\text { (w/v\%) }\end{array}$ \\
\hline $\mathrm{C} 1$ & $1: 2$ (PLGA65:35) & 10 & 10 & - \\
C2 & $1: 2$ (PLGA 50:50) & 10 & 10 & - \\
C3 & $1: 2$ (PLGA 50:50) & 10 & 10 & - \\
C4 & $1: 1$ (PLGA 50:50) & 20 & 20 & - \\
D1 & $1: 1$ (PLGA50:50) & 10 & - & 5 \\
D2 & $1: 1$ (PLGA50:50) & 15 & - & 5 \\
\hline
\end{tabular}

"C" refers to composite microsphere, "D" refers to double-walled microsphere. $\mathrm{C} 2$ and $\mathrm{C} 3$ are different in that, for $\mathrm{C} 2$, the drug was loaded into PLGA phase and for C3, the drug was loaded into PLLA phase during fabrication procedure.
Table 4

Encapsulation efficiency and particle size distribution

\begin{tabular}{llllll}
\hline Sample & $\begin{array}{l}\text { Encapsulation } \\
\text { efficiency }(\%)\end{array}$ & $\begin{array}{l}\text { Particle size } \\
(\mu \mathrm{m})\end{array}$ & $\mathrm{C} \mathrm{( \% )}$ & $\mathrm{N}(\%)$ & $\mathrm{O}(\%)$ \\
\hline $\mathrm{C} 1$ & $64.5 \pm 2.2$ & $308.9 \pm 213.3$ & 33.49 & 2.01 & 31.83 \\
$\mathrm{C} 2$ & $54.1 \pm 2.2$ & $307.3 \pm 185.6$ & 36.84 & 2.30 & 34.54 \\
$\mathrm{C} 3$ & $52.5 \pm 2.4$ & $305.1 \pm 180.4$ & 32.38 & 1.59 & 30.79 \\
$\mathrm{C} 4$ & $65.1 \pm 7.2$ & $771.9 \pm 320.9$ & 42.09 & 1.32 & 40.77 \\
$\mathrm{D} 1$ & $17.9 \pm 2.2$ & $116.9 \pm 62.7$ & 34.77 & 1.91 & 32.86 \\
$\mathrm{D} 2$ & $26.8 \pm 2.1$ & $143.0 \pm 72.8$ & 32.64 & 1.76 & 30.88 \\
\hline
\end{tabular}

XPS data for microspheres. Relative mass percentages of each compound on the surface.

Gentamicin content using UV-Vis spectrophotometer (Shimadzu, PC-3101, Japan) or high-performance liquid chromatography. Derivatization is done with $o$-phthaldialdehyde, and absorbance wavelength is $339 \mathrm{~nm}$ for UV-Vis spectrophotometer [29 30]. $O$ phthalaldehyde is used for derivatization in the case of HPLC. The mobile phase is $4.5 \mathrm{~g}$ of 1 -heptane sulphonic acid sodium salt in 11 of the liquid ( 800 $\mathrm{ml}$ methanol, $20 \mathrm{ml}$ of acetic acid and $180 \mathrm{ml}$ water). A Shimadzu 10 ADvp system with a workstation is used. Excitation and emission wavelengths were 332 $\mathrm{nm}$ and $445 \mathrm{~nm}$, respectively. A Lichrosorb RP 18 column of $250 \times 4 \mathrm{~mm}$ in size from Merck is used for separation (Tables 1-3).

Microspheres were suspended in $1 \%(\mathrm{w} / \mathrm{v})$ of Tween 80 in deionised water and sonicated in order to prevent particle aggregation. Approximately $50 \mathrm{mg}$ of the microspheres is required for the analysis. Particle size was analyzed using a Coulter laser diffraction particle size analyzer (Beckman coulter, Coulter LS 320, Small Volume module, CA, USA; Table 4).

The surface chemistry for the microspheres formed was analyzed using an X-ray photoelectron spectroscopy (XPS; Kratos Axis, Kratos Analytical, NY). It uses soft (200-2000 eV) X-ray excitation to examine the $\mathrm{C}, \mathrm{N}$ and $\mathrm{O}$ present on the surface for up to $5 \mu \mathrm{m}$ in depth. Nitrogen is present in only Gentamicin, and hence, we can relate this to the amount of drug present on the surface (Table 4).

\subsection{Characterization}

The microspheres, which are fabricated, are to be tested so as to check if double-walled microspheres are formed. The following procedures are adopted to 
do the sectioning of the microspheres and analyze the composition. A Leica Cryostat CM 1900 was used to cross-section the microspheres at $-20{ }^{\circ} \mathrm{C}$ in a tissuefreezing medium. The cross-sectioning was done at 30 $\mu \mathrm{m}$. The samples are loaded on to a SEM stud, and the cross-section is analyzed (Scanning Electron Microscope, Joel, JFC-5600 LV, Japan) after a platinum coating. Microspheres were cross-sectioned similarly and mounted onto gold slides and analyzed using an FTIR-microscope (Bio-Rad UMA 500) connected to FTIR spectrophotometer (Bio-Rad FTS-3500 ARX). The wave number used was from 1300 to $1500 \mathrm{~cm}^{-1}$ at a resolution of $2 \mathrm{~cm}^{-1}$. Ten points are randomly selected for analysis to get the transmission spectrum. The spectrum was compared against a single polymer for analysis.

\subsection{In vitro release}

Triplicate samples of $10 \mathrm{mg}$ of microspheres were suspended in $20 \mathrm{ml}$ phosphate buffer saline (PBS) in Nalgene centrifuge tubes. The tubes are placed in a bench top water shaker bath at $120 \mathrm{rpm}$ and $37^{\circ} \mathrm{C}$ (Cetomat WR, B. Braun Biotech International, Germany). The samples are drawn at predetermined time intervals after centrifugation (Eppendorf $5810 \mathrm{R}$ ) at 12,000 rpm for $3 \mathrm{~min}$ and replaced with fresh PBS. In the case of discs, the samples were collected directly, as no centrifugation is required.

\subsection{Degradation study}

For degradation studies, $30 \mathrm{mg}$ of microspheres is suspended in $20 \mathrm{ml}$ PBS and kept under similar conditions as that for in vitro study. At predetermined time intervals, the samples are drawn out and studied using Differential Scanning Calorimeter (DSC) and SEM. Thermal analysis of the microspheres was performed by means of a Modulated Differential Scanning Calorimeter (DSC 2920, TA instruments, USA). The samples were subjected to heating from 20 to $200{ }^{\circ} \mathrm{C}$ for the first heating ramp, cooled to $-10{ }^{\circ} \mathrm{C}$ and finally reheated to $200{ }^{\circ} \mathrm{C}$ on the second heating ramp, all at the rate of $10{ }^{\circ} \mathrm{C} / \mathrm{min}$. The data from the second heating ramp were used, and changes in glass transition temperature $\left(T_{\mathrm{g}}\right)$ and melting point $\left(T_{\mathrm{m}}\right)$ were studied with time in order to analyze the extent of degradation of polymer.

\section{Results and discussion}

\subsection{Part A: in vitro release from different types of discs and modified discs}

Implantable discs made by compressing the microspheres are prepared for the study. The study was directed towards the preparation of discs, as it was observed that the microspheres have released all of the Gentamicin in less than a day (results of release from microspheres are not shown). It appears that almost all of the Gentamicin remains close to the surface of the microspheres, and hence, it is released in less than a day. It can also be inferred that the size of the particles may play a larger role in the release of Gentamicin. Varying the fabrication conditions, like temperature, flow rate of air and aspirator ratio, will only change the size of the microspheres by a fraction of a micrometer or a few micrometers, and hence, the release can be expected to be similar.

The microspheres made of different types of PLGA are compressed into a 5-mm discs, and the in vitro release is studied. It was observed that the discs gave a biphasic behavior. There was an initial release observed which can be attributed to the release of Gentamicin from the microspheres that are present close to the surface when compressed. Then there was a lag phase observed as a result of no Gentamicin being released from the disc. It appears that the disc remains largely impermeable to water, and thus, there is no release of Gentamicin. The lag phase was followed by a second release, which can be attributed to a stage where the bulk degradation of the polymer reaches a critical level, allowing for the Gentamicin to diffuse out of the disc. It is known that PLGA undergoes bulk degradation [31,32].

PEG modified discs are prepared with an intention of modifying the porosity of the discs. It appears that by modifying the porosity of the discs made of PLGA microspheres, we will be able to modify the lag phase and the amount of initial release of Gentamicin. Hence, polyethylene glycol (PEG), which is a watersoluble polymer, is added to the PLGA solution before spray drying in order to achieve uniformity. It is anticipated that PEG will diffuse out of the discs leaving some pores, and Gentamicin will come out through pores, giving a larger initial release followed by the elimination of the lag phase. 
In the study, the effect of PEG content on the release profile was investigated. The effect of molecular weight of the PEG used was also investigated. It was observed that PLGA 50:50 releases Gentamicin faster (results/figure of in vitro release from pure discs is not shown) and degrades faster, and hence, for all the study of the modification of the discs, PLGA 50:50 is used as the main polymer.

The SEM pictures (data not shown) have shown increased agglomeration when PEG is added to PLGA, and hence, it was not possible to calculate the particle size of the particles formed. Also, the particles are not being used directly for in vitro release, and as discussed in the first paragraph on implantable discs, small change in the size of the particles does not change the shape of the release curve. It can be said that the particles lose their identity when they are compressed to form a disc. Hence, in the process of spray drying, the Gentamicin solution along with the polymer solution is mainly to achieve uniform product in terms of microparticles, which ensures a uniform distribution of Gentamicin and polymer in the disc.

The results (Fig. 1) show that adding PEG to PLGA and compressing the microparticles into discs will only change the release by changing the initial release and changing the position in time where the second release starts. However, the shape of the release curve remains largely of the same type, which again is a biphasic behavior. It was observed that adding about $2-5 \%$ PEG does not change the profile much as compared to the pure PLGA discs, and there is a small change in initial release. However, when about $10 \%$ of PEG is added, the initial release of Gentamicin largely increases. When the content is increased further, the initial release increases greatly, and almost the entire drug comes out in the initial release itself. It was also observed that the small increase in the height of the disc does not change the shape of the release curve significantly (when the mass of disc is increased from 25 to $35 \mathrm{mg}$ to get a disc of different thickness).

It can be observed that the initial release increases as the content of PEG increases. It can also be seen from the SEM pictures (Fig. 2F) that the addition of PEG to PLGA gives pores in the disc (the microparticles of PEG-PLGA are compressed into discs). Hence, it can be said that the addition of PEG
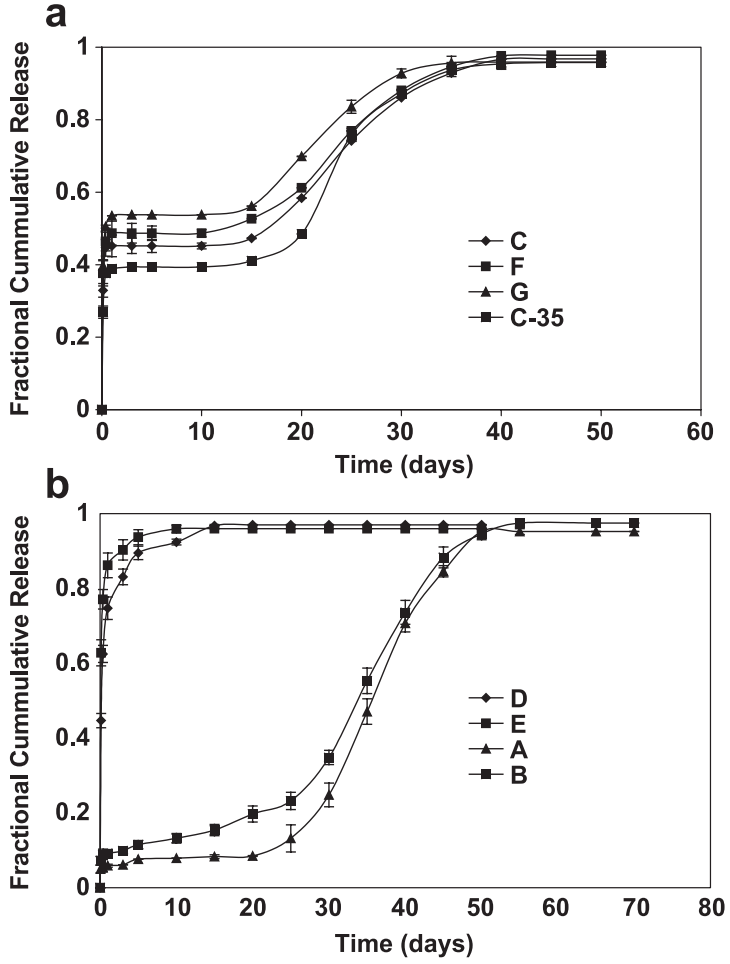

Fig. 1. (a) In vitro release from PEG-PLGA discs (notation is same as Table 1). C-10\% PEG (mol. wt. 8000); F-10\% PEG (mol. wt. 3350); G-10\% PEG (mol. wt. 1450). For all samples, PLGA 50:50 was used as the main polymer along with PEG. Mass of all discs is $25 \mathrm{mg}$ except for C-35, it is $35 \mathrm{mg}$. (b) In vitro release from PEGPLGA discs (notation is same as Table 4). A-2\% PEG (mol. wt. 8000); B-5\% PEG (mol. wt. 8000); D-15\% PEG (mol. wt. 8000); E-20\% PEG (mol. wt. 8000). For all samples, PLGA 50:50 was used as the main polymer along with PEG. Mass of all discs is $25 \mathrm{mg}$.

increases the initial release. It was also observed that the smaller the molecular weight of the PEG added, the higher is the initial release. However, it appears that there is not much effect in terms of where the second release starts. It is observed that the second release starts earlier than that of the pure PLGA microparticle-compressed discs. During the in vitro study, it was observed that the discs start to change their color and become transparent. This is the time when PEG tends to come out of the disc rapidly or when the time the PLGA starts to degrade largely. It is more likely that the PEG starts to come out of the disc before PLGA starts to actually degrade more, and that can be seen from the SEM picture of PEG-PLGA discs after 20 days of in vitro release (the SEM 

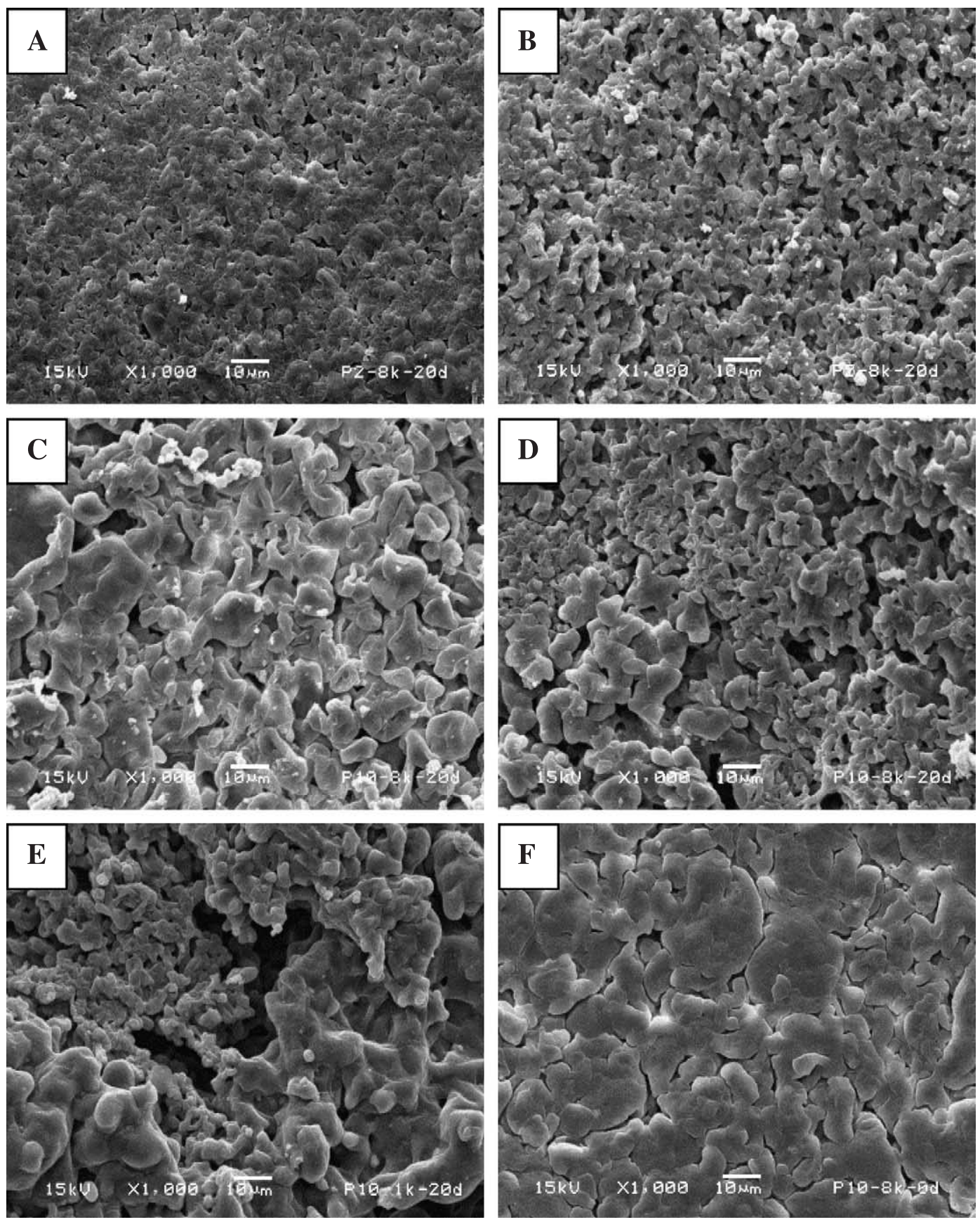

Fig. 2. Degradation of PEG-PLGA discs after 20 days compared to day 0. A-2\% PEG (mol. wt. 8000); B-5\% PEG (mol. wt. 8000); C-10\% PEG (mol. wt. 8000); D-10\% PEG (mol. wt. 3000); E-10\% PEG (mol. wt. 1000); F-10\% PEG (Mol. wt. 8000). A-E are after 20-day release and $\mathrm{F}$ is before in vitro study (refer to Table 1 for description of symbols). 
picture of PEG-PLGA shows more pores). The relative porosity after a 20-day release can be observed in Fig. 2.

Methylcellulose formed another polymer that was used along with PLGA to prepare microspheres with an intention of investigating the effect of possible change of porosity and polymer type on the release characteristics. Methylcellulose is not soluble in ethyl acetate, and it does not form a suspension either. Hence, dichloromethane was used in the fabrication of methylcellulose-PLGA microparticles. When dichloromethane is used, methylcellulose forms a turbid solution. It may be due to the fact that only the methyl-side group tends to dissolve in solution, whereas the cellulose backbone remains suspended hence giving a turbid look. As it does not form a uniform solution, the uniformity of the microparticles formed by using methylcellulose may not be high.

It can be seen in the release profiles (Fig. 3) from the methylcellulose-PLGA discs where the release tends to be very much fluctuating. This can be attributed to the fact that there is large nonuniformity. The figure shows that the release becomes much faster as the amount of methylcellulose is increased, which is similar to that when PEG is added. The profile is acceptable; however, since there is the possibility of large-scale nonuniformity, reproducibility is a cause for concern.

Calcium phosphate blended discs are prepared and tested whether they are successful in the modification of the porosity so as to achieve a release time of about

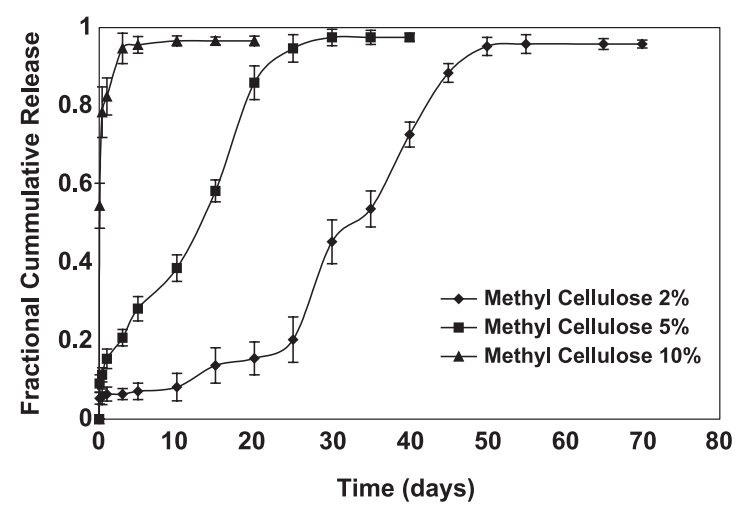

Fig. 3. In vitro release from methylcellulose-PLGA discs. For all samples, PLGA 50:50 was used as the main polymer.
6 weeks and an acceptable release profile. Hydroxylapatite and beta-tricalcium phosphate are the boneforming biomaterial. In order to prepare the discs, the microspheres were prepared from pure PLGA and are then blended with the calcium phosphate in the required ratio and the in vitro release is studied. Hydroxylapatite is a much better biocompatible material compared to beta-tricalcium phosphate, but it is relatively difficult to compress it into a disc as the surfaces and edges tend to break. Hence, the study of the calcium phosphate discs was largely related to using beta-tricalcium phosphate.

It was observed from the in vitro release data that when small quantity of the order of $10 \%$ of the total weight of the disc is the calcium phosphate, then all of the Gentamicin comes out very fast (results/figure not shown), and it appears that the porosity becomes very large, and the discs give a similar release to that of the microspheres. The amount of the calcium phosphate was later reduced to about $60 \%$ of the total weight of the disc, and it was observed that the release profile could be modified to an acceptable one (Fig. 4). However, the time of release remains to be about 2 weeks, which is still very short compared to the required release time of 6-8 weeks. Here, it can be observed that the hydroxylapatite-blended discs give a haphazard release profile, which can be attributed to the nonuniformity and surface roughness that was discussed before. It is now apparent that the reduction in the calcium phosphate content can actually reduce the porosity of the disc and hence release the Gentamicin relatively slowly. It was also observed that there is not much effect of the type of the PLGA that was used to prepare the microspheres.

As hydroxylapatite is difficult to be compressed into discs, only tri-calcium phosphate was used for the remaining study. As it can be seen that the calcium phosphate content determines the release time, the content of the calcium phosphate was further reduced in order to reduce the porosity of the disc. By doing so, it was observed that the release profile shifts to a biphasic behavior when there is an initial release followed by a lag phase, which is followed by a second release, and this is similar to the PEG-PLGA discs. Hence, it may be said that the content of the additive can only be above a critical value below which the release is biphasic. It was observed during the process of in vitro studies that the lesser the 

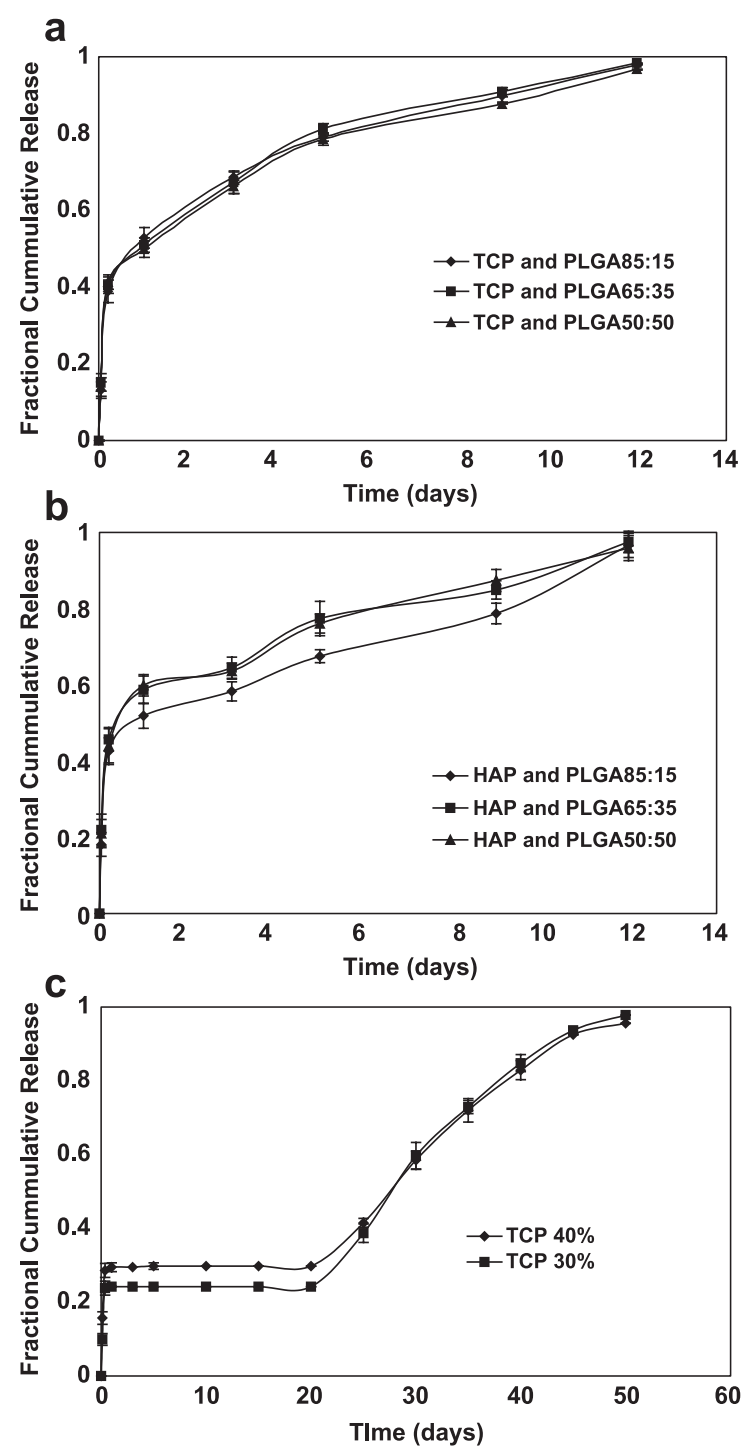

Fig. 4. (a) In vitro release discs made by blending beta-tricalcium phosphate (at 60\%) and microspheres. TCP is beta-tricalcium phosphate. (b) In vitro release discs made by blending hydroxylapatite(at $60 \%$ ) and microspheres. HAP is hydroxylapatite. (c) In vitro release from beta-tricalcium phosphate and microspheres blended discs at $40 \%$ and $30 \%$ calcium phosphate with PLGA 50:50 microspheres. T-beta-tricalcium phosphate.

content of the calcium phosphate that is added to the disc, the more it retains its shape to that of the original and vice versa, and this is due to the porosity and the ease of water uptake. Also, the blending process of the microspheres and the calcium phosphate have a disadvantage in that it is a mechanical process and uniformity at all times may not be achieved, and this is a cause for concern.

\subsection{Part $B$ and $C$ : formation of double-walled-composite microspheres and characterization}

In an effort to achieve better encapsulation efficiency and controlled release characteristics for a hydrophilic drug like Gentamicin, we understand that the size of the particles should be large and also the effect of water phase during the fabrication process should be minimized. In order that the size of the particles to be large, we have worked by taking high concentration of polymer solution, as the main factors influencing the formation of microspheres would be the concentration, stirring speed and the concentration of polyvinyl alcohol (PVA) solution to a lesser extent. Also, when the concentration of the polymers is very high, there will be immiscibility, which forms the basic for our fabrication. In order to avoid the diffusion of Gentamicin into the water phase during the fabrication by solvent evaporation method, we have worked on the fabrication of double-walled microspheres with a view of encapsulating the drug in the inner core and hence protecting it from the water phase.

The formation of microspheres while using a single solvent as in part B can be described by the spreading coefficient theory. Two polymers will become immiscible when the concentration exceeds a certain critical value, which is obtained by the phase diagrams. There are a variety of polymers with varying molecular weight, which makes it difficult to start from obtaining the phase diagram and then proceeding for the fabrication with the required concentration. Hence, a trial and error procedure is adopted. Previous studies have shown that double-walled microspheres can be produced when the concentration of the polymers is in the range of $15(\mathrm{w} / \mathrm{v}) \%-20(\mathrm{w} / \mathrm{v}) \%$. Handling of the samples at such a high concentration becomes extremely difficult as viscosity increases, which influences mixing, core and shell formation. This nonuniformity is a cause for concern. Hence, we have selected a concentration that is lower than the above in order to facilitate for better handling and have tried the possibility of producing double-walled microspheres.

For the case of part C, the property of PLLA not being soluble in ethyl acetate is utilized. PLLA 
solution in DCM containing Gentamicin as solid is poured into a PLGA solution in ethyl acetate. When a higher concentration of PLLA is poured into a lower concentration of PLGA, drops of PLLA will be formed around which PLGA will be distributed. During the hardening of microspheres, it was observed that, at the instant the drop is formed, it is still not hard but is soft. The DCM in semisolid droplets/particles tries to migrate radially outside, and hardening first occurs at the interface of PLLA (core)PLGA (shell). Now, at the interface, PLLA is totally solid, and PLGA is in dissolved state in EA. Again, DCM tries to come out radially in the outward direction, and EA tries to go inside. Along the path of DCM, PLLA tries to go to the outside, and along the path EA travels, PLLA solidifies. A stage reaches where the core has only EA for concentration balance and DCM totally diffused outside into EA. At this stage, PLA is entirely solidified inside, and the PLGA is in a dissolved state in surrounding EA. When this is poured into a water phase, the solvent will diffuse out, leaving a crater inside. The formation of the composite can also be explained by a similar hypothesis, removing EA and its diffusion into the core in the discussion. Since there is only water, it is difficult for it to travel inside evenly, and uneven craters are observed. Different concentrations have been worked on, and only those that successfully gave doublewalled microspheres and better encapsulation efficiency are presented.

The results from the FTIR-microscope (Fig. 5) show that PLLA gives a clear plot with two peaks, one around $1400 \mathrm{~cm}^{-1}$ and the other around 1475 $\mathrm{cm}^{-1}$. PLGA, however, does not give very clear peaks but has many crests and troughs. Hence, we can correlate the predominant crests and troughs to those of PLLA and the minor crests and troughs to be that of PLGA. For the case of part B, the results can be
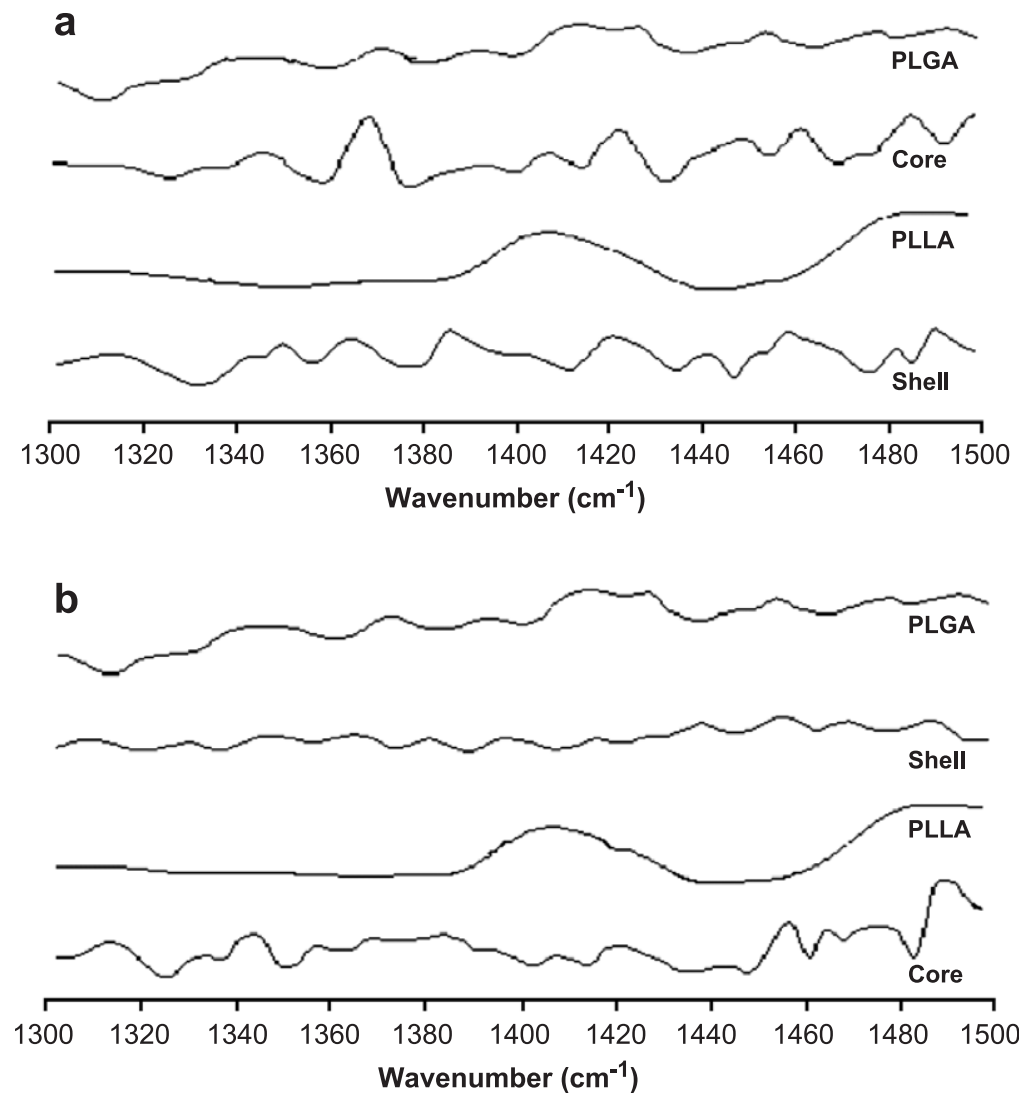

Fig. 5. (a) FTIR of C4. (b) FTIR of D1 [33]. 
interpreted thus, as the core is a mixture of PLGA with small quantity of PLLA and the shell is PLLA with small quantity of PLGA. Hence, we cannot exactly say that they are purely double-walled microspheres but are double-walled microspheres tending towards composites. It clearly shows that we have not reached the critical concentration that is required for immiscibility, and hence, we have to choose between easier handling and pure double-walled formation, depending on the requirements. For part $\mathrm{C}$, it can be observed that the shell is pure PLGA, and the core is a mixture of PLGA and PLLA. Hence, the microspheres from part $\mathrm{C}$ are more like double-walled than those from part B (Figs. 5 and 6) [33].

\subsubsection{Encapsulation efficiency, particle size and surface chemistry}

It can be observed (Table 4) that encapsulation efficiency is related to particle size $(\mathrm{C} 1, \mathrm{C} 2, \mathrm{C} 3, \mathrm{C} 4$ being higher and D1, D2 being lower). The larger the particle size, the higher the encapsulation efficiency. This is because of the large concentration of polymer used. Since the amount of solvent is small, the polymer solidifies faster and the time available for water to have an effect of removing Gentamicin will be reduced. In the case of part B, after the two solutions of PLLA and PLGA are mixed and sonicated, the final concentration still remains to be high, and hence, the particle size is large. Also, as viscosity is also high at high concentrations, the particle size distribution tends to be wide. In part $C$, when concentrated solution of PLLA is poured into dilute PLGA solution and sonicated, the particles are more or less formed and tend to be smaller in size. In this case, the particle size is not much affected by the stirring in water phase, which is actually the case for part B. Surface analysis clearly shows that the amount of drug present on the surface of the microspheres is very small. This can be concluded, as nitrogen is only present in Gentamicin, which is an amino acid. This is clearly what can be expected, as all the Gentamicin that is close to the surface will diffuse out into the water phase, as Gentamicin is highly hydrophilic.

\subsubsection{In vitro release}

In vitro releases from both the types of microspheres have primarily shown a biphasic behavior (Fig. 7). All the samples start with an initial release, which lasts for approximately 2 days. This can be explained by the diffusion of Gentamicin, which is present close to the surface into the PBS phase. It is often described that the initial release is due to the possible uptake of water into the microspheres, and the expansion that occurs because of the water uptake. After the initial release is observed, there is a lag phase, which is a characteristic of the polymer used. The polymers used generally undergo bulk degradation. Hence, the release can be due to either diffusion or bulk degradation or both. In the case of composites, it can be observed that the drug close
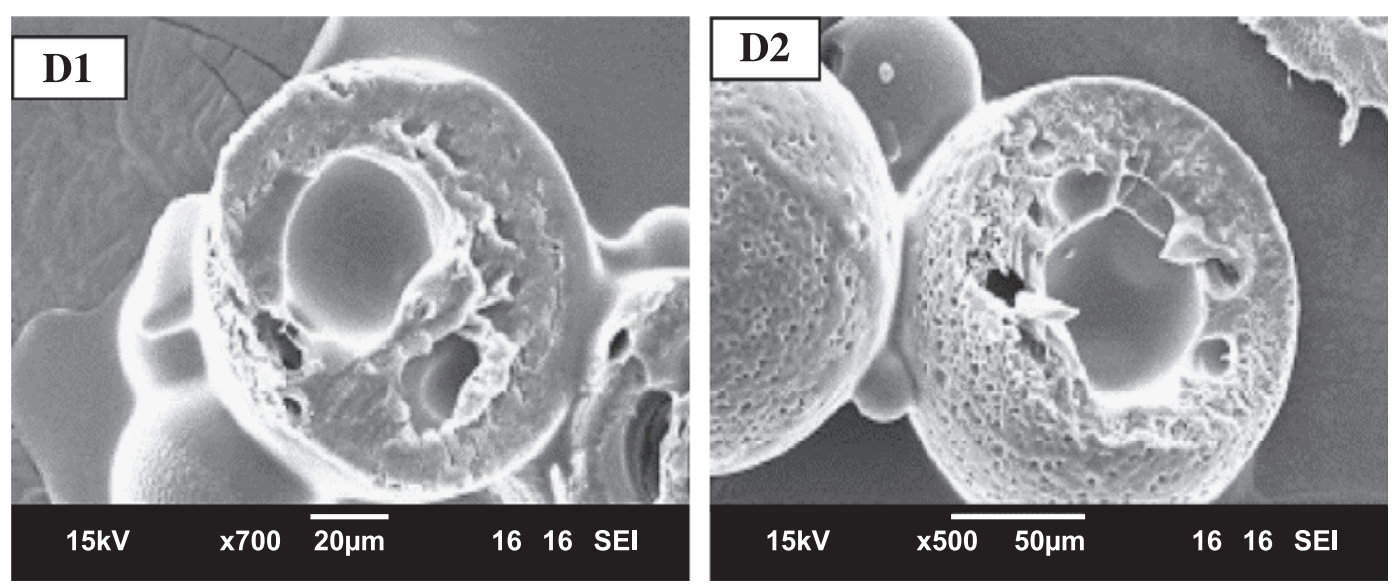

Fig. 6. SEM of cross-section of D1 and D2 (refer to Table 3 for description of symbols). 

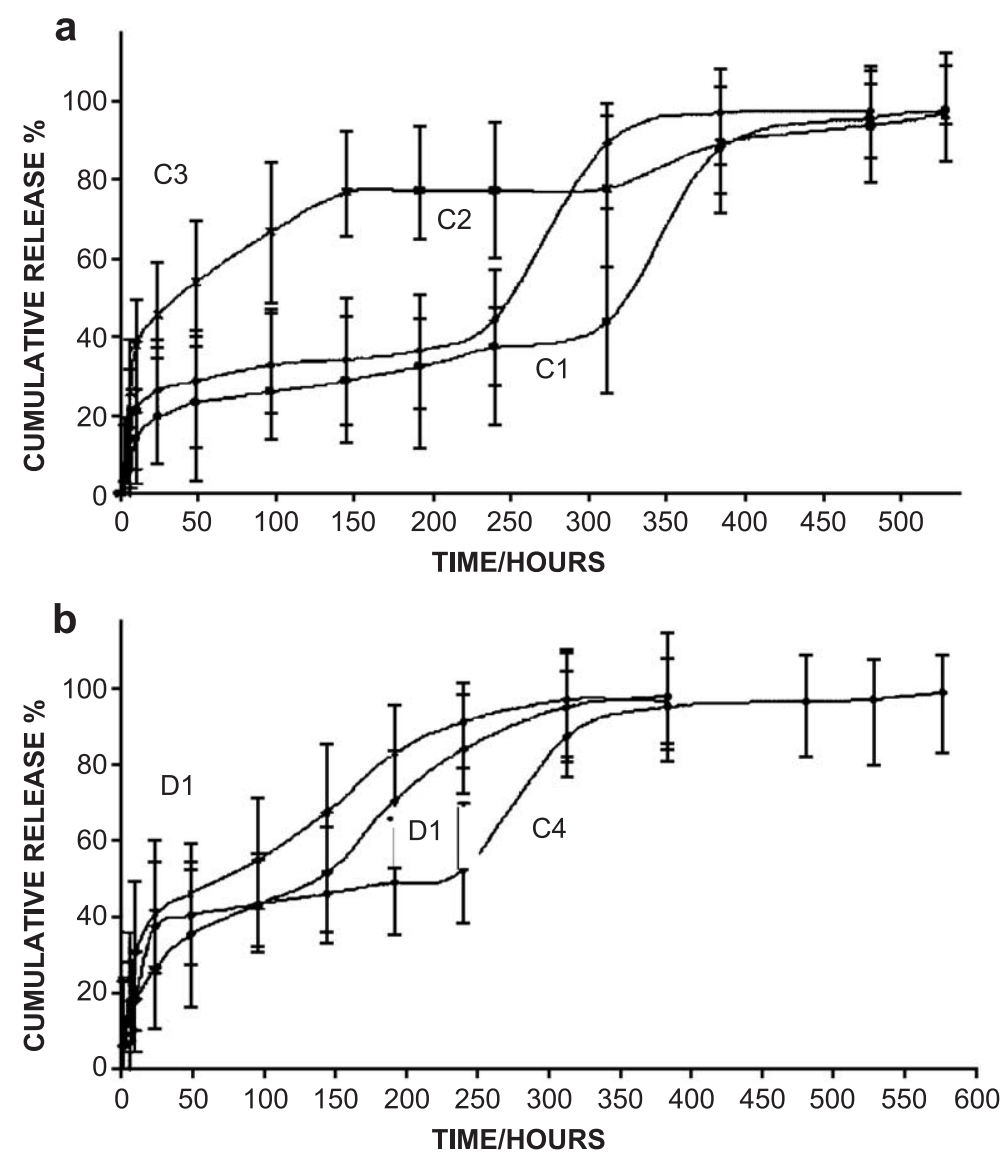

Fig. 7. (a) In vitro release for samples $\mathrm{C} 1, \mathrm{C} 2$ and $\mathrm{C} 3$ (refer to Table 3 for description of symbols). (b) In vitro release for microspheres D1, D2 and $\mathrm{C} 4$ (refer to Table 3 for description of symbols).

to the surface has diffused out in the initial period. It may be taken that the remaining drug is entrapped deep within the microspheres and comes out only when the polymer actually starts to degrade or after water has penetrated sufficiently into the microspheres. Hence, a lag phase is observed. However, drug release can still be observed during the lag phase, which can be attributed to the small-scale diffusion that might occur. In the case of double-walled microspheres, it is worthy to note that the lag phase is reduced and the release is faster compared to the composite microspheres. Taking the particle size into consideration, we can explain the results. For smaller particles, the release of Gentamicin by diffusion is easier, and for large particles, Gentamicin comes out when degradation starts. It can also be observed that, for $\mathrm{C} 2$ and $\mathrm{C} 3$, the difference in the process of drug loading (explained in the section on methods) changes the release profile. When drug is suspended in PLGA, the release is slower, and the release is faster when the drug is suspended in the PLLA phase, which can be expected, as PLLA generally tends to form the outer shell. It is possible that the release is influenced more by the size of the particles, where the release would be faster for smaller particles as diffusion plays a major role. For larger particles, there is a considerable lag phase, which is a characteristic of diffusion of the drug over long distance.

\subsubsection{Degradation study}

From the results of the degradation studies performed on various samples, it can be seen that 


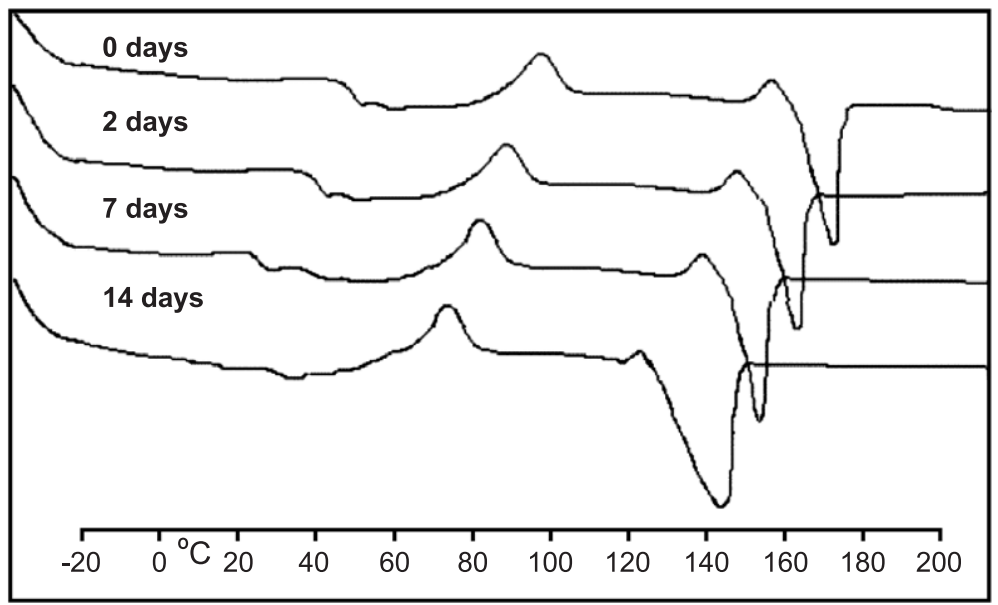

Fig. 8. DSC of D1 before, 2 days, 7 days, 14 days of in vitro release, (refer to Table 3 for description of D1).

there is no major change in thermal characteristics of the microspheres; however, there is a shift melting point and glass transition temperature towards lower temperature ranges (Fig. 8). The SEM pictures taken over a period of time show pores that are formed in the microspheres (Fig. 9). From the observed results, we can conclude that there is no possible bulk degradation in the microspheres during the period of study, but there is local degradation which can possibly due to the nonuniform structure of polymer in terms of molecular weight and copolymer ratios. Also, the Material Safety Data Sheet (Chemtrec, ANADA \#200-023) mentions that Gentamicin is stable up to around 100
${ }^{\circ} \mathrm{C}$, and the small crest at around $100{ }^{\circ} \mathrm{C}$ corresponds to the stability of the drug.

\section{Conclusions}

It is observed that the initial release from the compressed discs can be modified depending on the amount of additive, and choosing an appropriate type and amount of additive can eliminate the lag phase. However, the elimination of lag phase remains to be a major challenge. We were able to achieve double-walled microspheres for one of the two types of fabrication methods tried. Even when
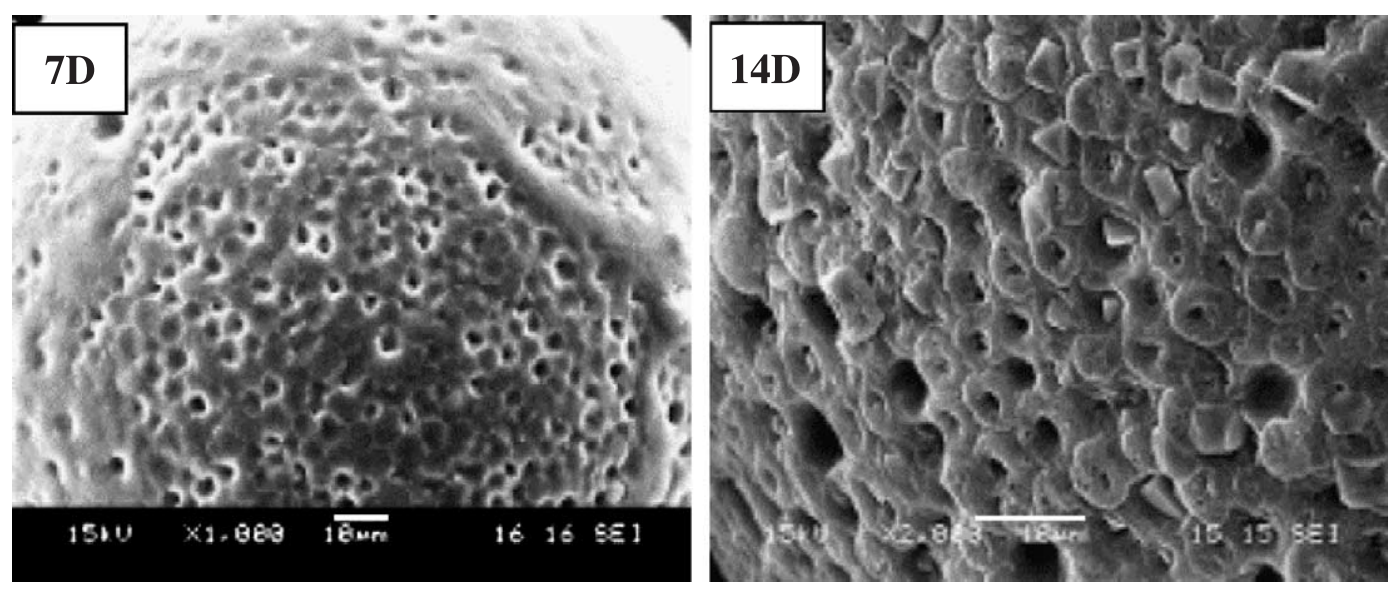

Fig. 9. Degradation of D1 at 7 days and 14 days (refer to Table 3 for description of D1). 
the other fabrication method did not give doublewalled microspheres, they have better encapsulation efficiencies and release curves. Since the fabrication is done with an intention of developing a biodegradable composition to treat Osteomyelitis, we can say that both the procedures have actually been successful in achieving better encapsulation efficiencies and release rate. However, further work needs to be carried out in order to extend the release time for microspheres and eliminate the lag phase for discs before in vivo studies are carried.

\section{Acknowledgements}

This work is supported by the National University of Singapore and Singapore-MIT Alliance under the grant numbers R279-000-095-112 and MEBCS project-003, respectively. We would like to thank Jian Jun Wang, Fangjing Wang, Lee Chian Guan Herman and Kwong Bing Fai for their technical assistance and many useful discussions through the project.

\section{References}

[1] K. Kanellakopoulou, E.J. Giamarellos-Bourboulis, Carrier systems for the local delivery of antibiotics in bone infections, Drugs 59 (6) (2000) 1223-1232.

[2] E. Sanchez, M. Baro, I. Soriano, A. Perera, C. Evora, In vivo-in vitro study of biodegradable and osteointegrable Gentamicin bone implants, Eur. J. Pharm. Biopharm. 51 (2001) 151-158.

[3] M. Baro, E. Sanchez, A. Delgado, A. Perera, C. Evora, In vitro in vivo characterization of Gentamicin bone implants, J. Control. Release 83 (2002) 353-364.

[4] I. Soriano, C. Evora, Formulation of calcium phosphates/poly DL lactide blends containing Gentamicin for bone implantation, J. Control. Release 68 (2000) 121-134.

[5] S. Prior, C. Gamazo, J.M. Irache, H.P. Merkle, B. Gander, Gentamicin encapsulation in PLA/PLGA microspheres in view of treating Brucella infections, Int. J. Pharm. 196 (2002) 115-125.

[6] M.J. Blanco-Prieto, C. Lecaroz, M.J. Renedo, J. Kunkova, C. Gamazo, In vitro evaluation of Gentamicin released from microparticles, Int. J. Pharm. 242 (2002) 203-206.

[7] G. Wei, Y. Kotoura, M. Oka, T. Yamamura, R. Wada, S.H. Hyon, Y. Ikada, A bioabsorbable delivery system for antibiotic treatment of Osteomyelitis, J. Bone Jt. Surg. 73-B (2001) $246-252$.
[8] W. Friess, M. Schlapp, Release mechanisms from Gentamicin loaded plga microparticles, J. Pharm. Sci. 91 (3) (2002) $845-855$.

[9] M. Changez, K. Burugapalli, V. Koul, V. Choudhary, The effect of composition of poly acrylic acid gelatin hydrogel on Gentamicin sulphate release in vitro, Biomaterials 24 (2003) $527-536$.

[10] J. Mauduit, N. Bukh, M. Vert, Gentamicin poly lactic acid blends aimed at sustained release local antibiotic therapy administered per operatively: I. The case of Gentamicin base and Gentamicin sulfate in poly DL lactic acid oligomers, J. Control. Release 23 (1993) 209-220.

[11] J. Mauduit, N. Bukh, M. Vert, Gentamicin poly lactic acid blends aimed at sustained release local antibiotic therapy administered per operatively: II. The case of Gentamicin sulfate in high molecular weight poly DL lactic acid and poly L-lactic acid, J. Control. Release 23 (1993) 221-230.

[12] J. Mauduit, N. Bukh, M. Vert, Gentamicin poly lactic acid blends aimed at sustained release local antibiotic therapy administered per operatively: III. The case of Gentamicin sulfate in films prepared from high and low molecular weight poly DL lactic acid, J. Control. Release 25 (1993) 43-49.

[13] K. Panduranga Rao, M. Sivakumar, Preparation, characterization and in vitro release of Gentamicin from coralline hydroxyapatite-gelatin composite microspheres, Biomaterials 23 (2002) 3175-3181.

[14] S.S. Sampath, K. Garvin, D.H. Robinson, Preparation and characterization of biodegradable poly L lactic acid Gentamicin delivery systems, Int. J. Pharm. 78 (1992) 165-174.

[15] I. Gursel, F. Yagmurlu, F. Korkusuz, V. Hascirci, In vitro antibiotic release from poly 3-hydroxybutyrate-co-3-valerate rods, J. Microencapsul. 19 (2) (2002) 153-164.

[16] I. Gursel, F. Korkusuz, F. Turesin, N. Gurdal Alaeddinnoglu, V. Hasirci, In vivo application of biodegradable controlled antibiotic release systems for the treatment of implant related Osteomyelitis, Biomaterials 22 (2002) 73-80.

[17] Y.Y. Huang, T.W. Chung, Microencapsulation of Gentamicin in biodegradable PLA and/or PLA/PEG copolymer, J. Microencapsul. 18 (4) (2001) 457-465.

[18] X. Zhang, U.P. Wyss, D. Pichora, A.F.A. Goosen, Biodegradable controlled antibiotic release devices for osteomyelitis: optimization of release properties, J. Pharm. Pharmacol. 46 (1994) 718-724.

[19] T.H. Lee, J.J. Wang, C.H. Wang, Double walled microspheres for sustained release of highly water soluble drugs: characterization and irradiation studies, J. Control. Release 83 (2002) 437-452.

[20] F. Wang, M. Eng Thesis, Department of Chemical and Environmental Engineering, National University of Singapore, 2001.

[21] K.J. Leach, E. Mathiowitz, Degradation of double walled polymer microspheres of PLLA and PCPP: SA 20:80: I. In vitro degradation, Biomaterials 19 (1998) 1973-1980.

[22] K.J. Leach, S. Takahashi, E. Mathiowitz, Degradation of double walled polymer microspheres of PLLA and PCPP: SA 20:80: II. In vivo degradation, Biomaterials 19 (1998) $1981-1988$. 
[23] K. Leach, K. Noh, E. Mathiowitz, Effect of manufacturing conditions on the formation of double walled microspheres, J. Microencapsul. 16 (2) (1999) 153-167.

[24] H.K. Lee, J.H. Park, K.C. Kwon, Double walled microparticles for single shot vaccine, J. Control. Release 44 (1997) $283-293$.

[25] A. Matsumoto, Y. Matsukawa, T. Suzuki, H. Yoshino, M. Kobayashi, The polymer alloys method as a new preparation method of biodegradable microspheres: principle and application to cisplatin loaded microspheres, J. Control. Release 48 (1997) 19-27.

[26] K.J. Pekarek, J.S. Jacob, E. Mathiowitz, Double walled polymer microspheres for controlled release, Nature 367 (1994) 258-260.

[27] K.J. Pekarek, J. Pekarek, J.S. Jacon, E. Mathiowitz, One-step preparation of double walled microspheres, Adv. Mater. 6 (9) (1994) 684-687.

[28] K.J. Pekarek, M.J. Dyrud, K. Ferrer, Y.S. Jong, E. Mathiowitz, In vitro and in vivo degradation of double walled polymer microspheres, J. Control. Release 40 (1996) 169-178.
[29] A. Cabanes, Y. Cajal, I. Haro, J.M. Garcia Anton, M. Arboix, F. Reig, Gentamicin determination in biological fluids by HPLC, using tobramycin as internal standard, J. Liq. Chromatogr. 14 (10) (1991) 1989-2010.

[30] D.H. Robinson, S.S. Sampath, Comparison of new and existing spectrophotometric methods for the analysis of tobramycin and other aminoglycosides, J. Pharm. Sci. 79 (5) (1991) 428-431.

[31] J. Sriramkamal, H.R. Dennis, A bioresorbable, polylactide reservoir for diffusional and osmotically controlled drug delivery, AAPS PharmSciTech 1 (4) (2000) (article 29).

[32] R.K. Agarwal, Development and evaluation of bioresorbable membranes for the controlled release of tetracycline $\mathrm{HCl}$ into intracrevicular fluid, PhD dissertation, Department of Pharmaceutical Sciences, College of Pharmacy, Omaha, NE, University of Nebraska Medical Center, 1994.

[33] P.K. Naraharisetti, M.D.N. Lew, C.H. Wang, Y.C. Fu, Double walled microspheres for the encapsulation of a hydrophilic drug, 30th Annual Meeting and Exposition of the Controlled Release Society, 19-23 July, Glasgow, Scotland, 2003. 